\title{
Patients' Description of Unexpected Interactions: A Critical Ethnography of the Quality of Doctor-Patient Interactions in One Educational Hospital in Shiraz, Iran
}

\author{
Ahmad Kalateh Sadati, ${ }^{1}$ Kamaran Bagheri Lankarani, ${ }^{2,}$ and Soroor Hemmati ${ }^{3}$ \\ ${ }^{1}$ Department of Sociology, Yazd University, Yazd, IR Iran \\ ${ }^{2}$ Health Policy Research Center, Shiraz University of Medical Sciences, Shiraz, IR Iran \\ ${ }^{3}$ Department of Education, Shiraz University of Medical Sciences, Shiraz, Shiraz, IR Iran \\ "Corresponding author: Kamaran Bagheri Lankarani, Health Policy Research Center, Shiraz University of Medical Sciences, Shiraz, IR Iran, E-mail: lankaran@sums.ac.ir \\ Received 2016 April 06; Revised 2016 June 26; Accepted 2016 July 14.
}

\begin{abstract}
Background: Sociologists regularly criticize the unbalanced doctor-patient interaction (DPI). A few previous studies have investigated DPI in developing countries.

Objectives: This study aimed to explore the nature of DPI in one educational hospital in Shiraz, Iran, according to the views of patients and their relatives.

Methods: This is a critical ethnography study that was conducted using a triangulation strategy in 2014, including156 clinical consultations, 920 hours of participant observations, and 6 focus groups with 51 patients and their families using a critical ethnography method. Sampling was purposeful, and the data were interpreted according to an exploration of the power relationship between the doctor and the patient in the context of the study according to the opinions of patients and their relatives.

Results: The study showed that participants faced unexpected interactions, which included four features: unequal, unprofessional, instrumental, and non-cooperative. Although this form of interaction was explored to examine the struggle between the voice of medicine and the voice of the lifeworld, the results showed that the DPI in the context of our study contains hegemonic features, which are related to physicians' autonomy.

Conclusions: These four themes revealed that patients are criticized about this form of interaction. These themes not only are not in line with the responsibilities and ethics of medicine but also are in contrast to it. It seems that patient-centered approaches can help solve the problem. In addition, the health system should monitor DPIs and promote them by reforming their structure.
\end{abstract}

Keywords: Doctor-Patient Interaction, Critical Ethnography, Iran

\section{Background}

Lifeworld has distinguishing signs and symbols (1) that facilitate social interaction. Therefore, an interaction is a transfer of meanings between two or more people with specific signs and symbols. The doctor-patient interaction (DPI) is a mutual relationship (2) that refers to the verbal communication that takes place between a physician and a patient during a medical consultation (3). The DPI also includes non-verbal communication, such as touch, facial expressions, and tone of voice, which are essential for a successful interaction (4). At the same time, both parties discuss the reason for the medical visit, such as a disease; this is also a time when they pass judgment on the quality of the interaction.

During the last five decades, DPI has become a dominant subject in many disciplines $(5,6)$. Sociologists, anthropologists, and medical researchers have had many debates about DPI. Among sociologists and medical researchers in Parsonian theory, a functional approach is central. In this view, the core phrase of Parsons, which is called the sick role, is the base, where a doctor plays a key role in the interaction with a dominant position. He/she performs an examination and then issues orders for the patient to follow (7). In this approach, the asymmetrical power relationship between the doctor and the patient is legitimized (8).

In contrast, they are anthropologists and critical sociologists. Critical medical anthropologists emphasize a culture of medicine (9), which reproduces capital. Thus, from their point of view, health and healthcare must be free of a capitalist economic system (10). In contrast, sociologists have criticized patient suppression during interactions with doctors. Habermas believed that modern medicine is an expert system that suppresses patients' lifeworld. For Foucault, DPI is a knowledge-power discourse that formed during the development of modern medicine beginning in the 18th century (11). According to these views, the quality of DPI is dependent on the doctor's power, which can 
lead to a distorted interaction.

Since an active DPI plays an important role in providing proper medical care, insight into the patients' view can enrich this relationship (12). Therefore, this study focused on the nature of DPI from the perspective of the patients and their families. As previously mentioned, doctors and patients evaluate the quality of the interaction. For example, a patient might observe whether the physician sympathizes with or is concerned about his/her needs. Thus, this study aimed to investigate how the DPI is evaluated by patients and their families. It explored the experiences of patients in their interactions with doctors as well as their expectations of these interactions.

In addition, while several similar studies have been carried out worldwide, no study has focused on the subject in Iran.

\section{Objectives}

Therefore, this study is necessary due to this lack any investigation into this issue and the importance of the subject.

\section{Methods}

This was a critical ethnography study that asked what could be in order to disrupt tacit power relationships and perceived social inequalities (13). Although an explanation of DPI can be created using either a non-critical or a critical method, we selected a critical method to explore the inequalities in relationships between doctors and patients. Therefore, this study was conducted at one educational hospital in Shiraz, Iran, from January to August 2014. We used triangulation methods to gather our data, which included the following:

1. Over 920 hours of participant observations were conducted in hospital wards. In this study, the researcher was a complete participant in observing DPIs. The researcher was also a clinical supervisor and therefore had to conceal his identity. During these observations, the researcher spoke with patients and their families and asked them about the doctors' interactions with them, specifically the patients who were dissatisfied with these interactions. Data that were included in this segment were purposeful and encompassed observation notes and patient concerns that were recorded in a written form.

2. Digital recordings were made of 156 clinical consultations from eight scientific faculty members (SFMs); these recordings were later transcribed. Here, a clinical consultation was a daily and routine visit made by the SFMs in the wards. Four cardiologists, three internists, and one neurologist allowed us to record the consultations.
3. Six focus groups were designed with patients and their families in five wards. For this reason, the conference hall of each ward was used for the interviews. All patients and their family members who had spent at least one night at the hospital were included in our study. Sampling was done purposefully; interviews were conducted with those who were ready for these discussions. Generally, our participant numbers were from 6-16 members per discussion (Table 1). Verbal consent was obtained from the participants and then recorded digitally and transcribed. The questions were as follows: What is your opinion about the relation and interaction of your doctor with you in this hospital? How do you evaluate this interaction? What are your expectations from the physicians? The characteristics of the focus groups are shown in Table 1.

All transcribed data were analyzed using a critical methodology based on a triangulation strategy. In this study, Carspecken's critical ethnography was used for the data analysis. Carspecken explored the power relation that he termed "reconstructive analysis." This method considers the cultural norms that shape people's behavior. The contexts that represent these values were interpreted. In this analysis, the participants' statements were analyzed according to three main validity claims: subjective, objective, and normative/evaluative. Subjective claims represent a patient's perception of an interaction. Objective claims represent certain objects and events that occur during the interaction. Normative/evaluative claims indicate judgments made by the patients and their families. Our method was based on Carspecken's reconstructive analysis (14). Simultaneously, the observer comments [OC] on the context was included to enrich the overall analyses and to add credibility to the claims. From the OC special circumstances were chosen for analysis. Meaning unit refers to participants' statements.

Validation was observed in three ways. A member's check was used as a tool for validation (15). In this process, the researcher' interpretation from his observations and focus group discussions were returned to the patients and their family members for verification. We also used a triangulation method to collect data for validation (16). Finally, during the study, the trustworthiness of the data analysis was ensured by three researchers. This study was based on the ethical codes of the American sociological association (17) and the Declaration of Helsinki about research ethics (18). Based on these considerations and the ethics of research, all names of the physicians and patients in this article are factious. In addition, in all parts of the study except the participant observation, we obtained the consent of the participants. About participant observation we mentioned the limitation. 
Table 1. The Focus Groups' Demographic Information

\begin{tabular}{|c|c|c|c|c|c|c|c|}
\hline & Ward & Number of Participants & Females & Males & Number of Patients & Patient Relatives & Duration of the Interview (Hours) \\
\hline $\mathbf{1}$ & Internal medicine & 6 & 2 & 4 & 5 & 1 & $1: 10$ \\
\hline 2 & Urology & 11 & 5 & 6 & 5 & 6 & $1: 28$ \\
\hline 3 & $\mathrm{CCU}$ & 16 & 7 & 9 & 13 & 3 & 1: 15 \\
\hline 4 & General surgery & 5 & 5 & 0 & 4 & 1 & $1: 20$ \\
\hline 5 & Cardiac surgery & 5 & 3 & 2 & 0 & 5 & $1: 12$ \\
\hline \multirow[t]{2}{*}{6} & General surgery & 8 & 1 & 7 & 7 & 1 & $0: 42$ \\
\hline & Total & 51 & 23 & 28 & 34 & 17 & 7:07 \\
\hline
\end{tabular}

\section{Results}

Our results showed that some patients and their families faced an unexpected type of interaction. Forms of interaction were unacceptable to them, which led to their disappointment. The main reason for their disappointment was a weakness in the DPI. They believed that the DPI was unacceptable due to the doctor's position. They felt that doctors should consider patients feelings and problems, interact appropriately, exhibit punctuality, and be responsible. They wanted the doctor to communicate better with the patients and give them a progress report. Some doctors not only ignored these obligations but also suppressed the patients and their families. Therefore, patients were faced with uncertainty with the DPI. In this scenario, they were cautiously worried about future interactions with the doctor during his/her upcoming visits.

The unexpected interaction refers to ignoring patients' rights. Even though patients know what is acceptable in a DPI, they seem powerless to claim this right. In this situation, patients can officially file a complaint, but it rarely happens. They often believe that if they complain and the doctor finds out, he/she may stop or reject their treatment. In this condition, having a good doctor with a good DPI is a random chance that it merely depends on a doctor's personality. The unexpected interaction included four themes: unequal, unprofessional, instrumental, and noncooperative.

\subsection{Unequal}

In an unequal interaction, one side of the interaction is lower than the other. In this type of interaction, doctors are in a dominant position. This situation allows them to determine all processes of the interaction, including how the interaction should start, continue, and finish. Therefore, patients and their relatives do not have any opportunity in the interaction. Their only opportunity to interact is to carry out the orders given by the physician.
4.1.1. OC

Due to the educational structure, interactions were merely between physicians and medical students. Hence, this form of interaction is very common on SFMs daily rounds. Patients and their relatives have many questions concerning their illness, but there is no clear response from SFMs. Even when they present their questions, the SFM's reply is often inadequate and sometime ambiguous, which creates real crises for the people involved. Hence, because of the asymmetrical power relationship, their questions are ignored by physicians. In contrast, it seems that SFMs do not visit patients every day and sometimes not for more than a week. In this situation, patients complain to the on-duty supervisor or hospital manager, but they do not complain to the doctor directly, which illustrates the asymmetrical power interaction. Since an unequal power interaction is defined as a norm in the context of this study, even medical students allow themselves to have bad interactions with patients.

\subsubsection{Specific Observation}

Mahin is a 22-year-old female who had an appendectomy two days ago. She has a newborn child and breastfeeds. This morning, she was told that she was going to be discharged from hospital. However, the resident surgeon did not visit her until 8 p.m. Her husband asked the nurse in charge to follow-up on the problem, but when the doctor became aware, he ignored the patient and did not visit her at all. He told the nurse, "The patient cannot be discharged. If she wants to go, she should complete the release sheet. Then she is allowed to go." Since the patient's perception was that she would be released but she was instead kept in the hospital for an unknown reason, the problem was forwarded to the supervisor in charge. When the resident did not provide an adequate reply, the issue was referred to the SFM by the supervisor to resolve the issue. The SFM called the resident, and the resident convinced the SFM that the patient should not be discharged clini- 
cally. However, the patient was forced to leave the hospital against medical advice with her own consent.

\subsubsection{Meaning Unit 1}

The patient does not dare to speak with his/her doctor because he/she is powerless. The patient is afraid to complain because the doctor may stop the treatment treat him/her badly. Thus, the patient does not allow himself/herself to complain in the presence of the doctor. Here, the doctor-patient relationship is like a master-servant relationship. The issue is not being afraid of the master. It is worse; it seems that the servant does not know his/her rights and does not allow himself/herself to even talk. Patients here are not aware of their rights (the patient's son has a renal problem).

\subsubsection{Meaning Unit 2}

The doctor-patient relationship in our country is a oneway street, and doctors do whatever they want.

Box 1. Horizon Analysis; Possible Validity Claims of the Quality of DPI Titled Unequal

\begin{tabular}{l}
\hline Claims \\
\hline Possible subjective claims \\
Quite foregrounded, quite immediate \\
The doctor orders and the patient should comply. \\
The patient does not complain. \\
\hline Less foregrounded, less immediate \\
\hline The patient is a passive party. \\
\hline High foregrounded, high immediate \\
Unequal interaction \\
Possible objective validity \\
\hline Quite foregrounded, quite immediate \\
\hline The patient is afraid to complain to the doctor. \\
\hline The doctor does whatever he/she wants. \\
\hline Less foregrounded, less immediate \\
\hline Master-servant interaction \\
\hline Possible normative claims \\
Less foregrounded, less immediate \\
Doctor should consider patients' rights. \\
Unequal interaction is not appropriate. \\
\hline
\end{tabular}

As shown in Box 1, the doctor-patient interaction is completely unequal. In this situation, the patient cannot do anything even when he/she feels that he/she is being ignored. The doctor can easily reject the patient and does not feel any responsibility towards his/her professional obligation.

\subsection{Unprofessional}

Unprofessional interaction refers to the passive interaction that takes place between doctors and patients in the same way that a medical student has inadequate information about the patients' past medical history. In this situation, the patient feels that the doctor does not have proper information about him/her. Thus, the patient thinks his/her health is not important to the doctor. When a doctor does not have comprehensive information about the disease, how can he/she provide appropriate treatment? This type of unprofessional interaction is a one-dimensional approach to diagnosis and treatment. For example, when a surgeon student merely focuses on the surgical problems of a patient and ignores all other aspects.

\subsubsection{OC}

The visits and consultations were conducted unprofessionally and were weak. The medical students obtained superficial information from the patients, and the SMFs solely relied on their reports. Specifically, during admission, the medical students focused on the part of the body that required treatment. For example, when a patient is admitted to the surgical ward, the doctor's history-taking only focused on his/her surgical problem. In this situation, other organs are ignored, particularly in patients with multiple problems. The issue becomes even more complicated when SFMs depend solely on this form of evaluation and treatment, as in consultation No. 87, a patient with cardiovascular problems (Table 2).

This consultation session revealed that the SFM and his resident did not have any conversation with the patient. Due to this unequal interaction, the patient did not have any opportunity to present his problem. Thus, only one organ was examined and treated, and the other organs were ignored. This method can lead to medical errors and malpractice as well as patient dissatisfaction.

\subsubsection{Specific Observer Comment}

Ali is a 25-year-old male who was admitted due to a renal stone diagnosis and was discharged from the urology ward after a few days because the lab data and ultrasound were normal. The patient was readmitted to an internal medicine ward due to general weakness with sepsis. He was afflicted with renal failure required dialysis. 
Table 2. DPI in Clinical Daily Visit No. 87

\begin{tabular}{ll}
\hline Participants & Statements \\
\hline SFM & Does he not have bradycardia? \\
\hline Resident & No, professor, he even had tachycardia. \\
\hline SFM & Has he been out of bed? \\
\hline Resident & Yes, professor. \\
\hline SFM & When was this electrocardiogram taken? \\
\hline Resident & $\begin{array}{l}\text { It was taken last night. I saw it, and there was } \\
\text { nothing. He is in good condition, and his lab report } \\
\text { does not show any problems. }\end{array}$ \\
\hline Nurse & Can he be discharged? \\
\hline SFM & It is unsafe until we know for sure. \\
\hline Resident & $\begin{array}{l}\text { Only with your permission because he has been } \\
\text { consuming Keflin for a long time. I have stopped } \\
\text { the order.(Finish) }\end{array}$ \\
\hline Time of consultation & \begin{tabular}{l} 
1:15 minutes \\
\hline
\end{tabular}
\end{tabular}

\subsubsection{Meaning Unit 1}

Doctors admitted him with a renal stone problem. On his discharge sheet, they wrote that he did not have any problems. However, his problem was not related to renal stones. Now he needs surgery for the insertion of a double lumen. Who should I complain to? (The patient's brother).

\subsubsection{Specific Observer Comment 2}

Hamid is a 32-year-old male who has been living with inflammatory bowel disease (IBD) and underwent a colostomy. He was referred to the hospital for an abscess. He expected that his surgeon would be an SFM, but the SFM forwarded the case to one of his residents. In the operating room, the resident asked Hamid, "Do you have a colostomy?"

\subsubsection{Meaning Unit 2}

"Why are you going to operate on me when you do not even know whether I have a colostomy or not? I have already told my doctor everything."

As Box 2 shows, patients and their families criticized the unprofessional interaction. An important point was that they understood the doctor had provided unprofessional care, which decreased their trust. This mistrust should be a warning sign for doctors. Due to the unequal power distribution, patients prefer not to complain.

\subsection{Instrumental}

Instrumental interaction refers to a form of interaction in which the doctor does not have any verbal or nonverbal communication with the patient. In this case, the doctor does not introduce himself/herself to the patient
Box 2. Horizon Analysis: Possible Validity Claims of the Quality of DPI Titled Unprofessionalism

\begin{tabular}{l}
\hline Claims \\
\hline Possible subjective claims \\
Quite foregrounded, quite immediate \\
The doctor does not spend time with the patient. \\
The doctor does not obtain a sufficient past history. \\
\hline Less foregrounded, less immediate \\
\hline The patient was readmitted due to the doctor's error. \\
\hline Possible objective validity \\
\hline Quite foregrounded, quite immediate \\
\hline His problem was not renal calculi. \\
\hline Just before the surgery, the resident asked Hamid “Do you have a colostomy?" \\
\hline Less foregrounded, less immediate \\
\hline To whom should I complain? \\
\hline Possible normative claims \\
\hline Less foregrounded, less immediate \\
\hline Doctors must also consider the patient's past history. \\
\hline Less foregrounded, less immediate \\
\hline An unprofessional interaction is not right. \\
\hline
\end{tabular}

and does not participate in any dialogue with the patient; a physical examination is rarely performed. In this condition the base of diagnosis and treatment is based on paraclinical data. For example, if a patient is referred for abdominal pain, a complete blood count (CBC) and abdominal ultrasound should be ordered. Thus, this instrumental interaction generally refers to passive relationship between doctor and patient which only relays on the patients' paraclinical data and not the physical exam or past history of illness.

\subsubsection{OC}

It is a routine behavior for the doctor not to introduce him/herself to a patient. Daily visits involve conversations between SFMs and their medical students and residents about the pathology and treatment (Box 1). The diagnosis and treatment were carried out using paraclinical data, such as magnetic resonance imaging (MRI), computed tomography (CT), laboratory data, and radiographs. The diagnosis and treatment were performed with the least possible amount of human interaction between the doctor and the patient. Thus, the patient thinks that his treatment is extremely mechanical. 


\subsubsection{Meaning Unit 1}

"The problem in this hospital is that rounds are done, but no one introduces him/herself to the patient. They do not even say, 'I'm your doctor.' Rounds are done, and all the patients' problems were tended to, but there was no interaction that allowed the patients to understand what is being done for them." (Maryam, a patient with a cardiac problem).

\subsubsection{Meaning Unit 2}

In the past three days, Dr. Fayez has visited our neighboring patient three times, but he did not introduce himself, and he did not communicate with the patient. He comes, reads a series of reports, gives a series of commands, and then leaves. Maybe the patient has a pain that needs to be reported? (The sister of a patient with a surgical problem).

\subsubsection{Meaning Unit 3}

"He comes and takes a look at the machine and then leaves. I mean, Dr. Farshad is not a doctor; he is an engineer, a mechanical engineer!"

Box 3. Horizon Analysis: Possible Validity Claims of the Quality of DPI Titled Instrumental

\begin{tabular}{l} 
Claims \\
\hline Possible subjective claims \\
Quite foregrounded, quite immediate \\
The doctor does not have a dialogue with the patient. \\
There is no introduction by the doctor. \\
The doctor does not do a physical exam. \\
Less foregrounded, less immediate \\
Only the doctor is physically present. \\
The doctor relies on paraclinical data. \\
Possible objective validity \\
Quite foregrounded, quite immediate \\
He did not introduce himself and did not communicate with the patient. \\
He is not a doctor! He is an engineer, a mechanical engineer! \\
\hline Possible normative claims \\
Less foregrounded, less immediate \\
Doctors must have a dialogue with patients. \\
Doctors must examine the patient. \\
Less foregrounded, less immediate \\
\hline Instrumental interaction alone is not right. \\
\hline
\end{tabular}

As the horizon analysis shows, the doctor-patient interaction is carried in an instrumental and mechanical fashion. The doctor only reads some reports, such as vital signs or paraclinical data, and uses these documents to prescribe an order. Most patients expect doctors to interact with them like normal human beings, not a device.

\subsection{Non-Cooperativeness}

Another feature of unexpected interaction is noncooperativeness, where a doctor or medical team makes a decision without the participation of the patient. This type of interaction can increase the possibility of medical errors. Additionally, in a non-cooperative interaction, the patient does not know what will happen to him/her; the patient is in limbo. Therefore, patients have a clear right to know the process of his/her treatment.

\subsubsection{OC}

Doctors do not communicate with the patient. They do not provide adequate information for their patients. Sometimes, if the patient has a question, they reply with short answers. It is a rare thing for a doctor to suggest various types of treatments to the patient or ask his/her opinion. In this situation, the patient is puzzled.

\subsubsection{Meaning Unit 1}

Doctors do not give us the right to participate in the treatment at all. We do not have any right to participate. I'm upset because I do not know what is going to happen to me. The doctor does not want us to present our opinion about our own treatment at all. (Zahra, a patient with a surgical problem).

\subsubsection{Meaning Unit 2}

If we have a question during the course of the disease, they do not provide a clear answer. For example, I asked my doctor what he was going to do. He just replied, 'I have to wait for fifteen weeks, then I will think of something.' I am completely confused!" (Maryam, who has multiple sclerosis and a surgical problem).

As the horizon analysis showed, the authoritarian model of interaction leads to patient dissatisfaction. They were upset about this type of interaction.

\section{Discussion}

The results of this study showed that interaction in this hospital was unexpected for both patients and their families. This unexpected interaction has four characteristics: unequal, unprofessional, instrumental, and non-cooperative. All of these themes indicate that DPI in these cases not only are not in direction of responsibilities and ethics of 
Box 4. Horizon Analysis: Possible Validity Claims of the Quality of DPI Titled NonCooperative

\begin{tabular}{l}
\hline Claims \\
\hline Possible subjective claims \\
Quite foregrounded, quite immediate \\
The doctor does not consider the patient's opinion. \\
Treatment is one way. \\
Less foregrounded, less immediate \\
The patient does not have any role. \\
Possible objective validity \\
Quite foregrounded, quite immediate \\
\hline The doctors do not give us the right to participate in the treatment at all. \\
They do not provide an obvious answer. \\
\hline Possible normative claims \\
\hline Less foregrounded, less immediate \\
\hline Doctors must consider the patient's opinion. \\
The doctors should consult with their patients. \\
\hline Less foregrounded, less immediate \\
Cooperative treatment is a right. \\
\hline
\end{tabular}

medicine but also are in contrast of it, specifically unprofessional and instrumental characteristics. They do not have a clear understanding of the treatment process and also cannot predict the doctors' reaction. Despite the fact they know that the asymmetrical power reaction is not an appropriate interaction, they cannot do anything about it. Doctors do not participate in any active conversation with patients; they do not even allow them to participate in the interaction. This shows how doctors ignore their professional duties and ethics where patients are concerned.

The results of other studies have confirmed our findings. According to Mishler, medicine has its own voice, which is in contrast to the lifeworld voice of patients. The medical voice also has its own specific terminology that uses physical symptoms descriptions, which are based in a biomedical model, which focuses on a technical explanation of the body. This technical interest is incompatible with the patients' lifeworld. Because patients' lifeworld is a lived experience that is dependent on interaction, this instrumental form does not satisfy them (19). A biomedical model has also been successful in practice; hence, it has dominated patients' life world. Theory of Mishler can explain three themes of our findings: unequal, noncooperative and instrumental. On the other hand, studies of Silverman (20) Atkinson (21) and Barry et al. (22) showed that medicine has more than one voice. However, it is unimportant to determine how many voice does medicine have; the only important point is that medicine has an instrumental and technical voice, which is not acceptable by the patient's lifeworld. In the past four centuries, this voice has been successfully developed in a manner to allow it to become a dominant voice. Unequal, non-cooperative, and instrumental characteristics are related to the domination factor.

In continuation, DPI in our study was heavily dependent on modern medicine discourse, which is phrased in Haberma's view as expert discourse (10) and lauded in Foucult's view as medical knowledge-power discourse (11). Therefore, the quality of DPI is entirely dependent on the quality or grade of discourse. In any DPI, if the discourse is more dominant, the patient's dissatisfaction will be greater, which is directly related to the existing asymmetrical power relationship. The patients in our study realized that the doctors' interactions with them were unprofessional. However, a majority of them did not file a complaint. This shows the hegemonic feature of DPI, which suppresses the patient in the worst way. A big part of the reason goes back to the formation of modern medicine discourse.

In the context of our study, one of the reasons for this type of DPI is a weakness of the medical structure. Although unequal, non-cooperative and instrumental are the general characteristics of medicine in the world, but unprofessional interaction is unacceptable and needs to be further discussed. The question also remains why these physicians approach treatment by neglecting some observation protocols? It seems that this action is partially related to underlying weakness in the medical structure to control and supervise their behavior, so instead, they do as they please. Due to this situation, the diverse forms of domination and suppression were identified in this context (23, 24). We suggest further study in order to determine why this occurs.

Finally, there are two forms of doctor-patient relationships: socio-emotional and task-oriented. In the socioemotional model, there is intrinsic communication, interpreted affect, linking, and a satisfactory relationship between the doctor and patient. A task-oriented model involves question-asking, information-giving, counseling, management, and treatment approaches by doctor and question-asking, full, accurate report of medical symptoms and history approach by patient (25). Our findings showed that DPI in the context of our study was neither similar to these two types. There was no active questionasking communication or other features which were mentioned above. In the study of Barry et al. (22), it was shown that the quality of interaction is dependent on the quality of using voice of medicine or the lifeworld. Our findings indicated that the voice of the lifeworld did not have 
any place in DPI. However, because good doctor-patient interactions are closely related to quality of care (26), policymakers should pay attention to the promotion of DPI. In this approach, a patient-centered model can be effective (27).

\subsection{Conclusion}

In the context of our study, DPI contains a hegemonic feature that is unacceptable for patients. In this situation, the interaction is unequal, unprofessional, instrumental, and non-cooperative. Therefore, the patients' lifeworld is suppressed by the doctor-centered condition. Patients do not have a clear understanding of interactional processes. The interaction is completely dependent on the personal behavior of the doctor and not his/her professionalism. Because DPI has an important role in medical care, policymakers must therefore establish suitable structures based on patient-centered approaches. Further qualitative and quantitative studies are suggested.

\subsection{Limitations}

The biggest limitation was concerning participant observation. Because the researcher hid his role as researcher, it was a problematic situation based on ethical issue. However, we did not have an alternative approach to gather these data. Another limitation was that even though the severity of the patient illness and disease may have had an effect on the DPI, we could not categorize the patients according to their severity of illness.

\section{Acknowledgments}

This research was extracted from the Ph.D. dissertation of Ahmad Kelateh Sadati in sociology entitled "critical narrative analysis of doctor-patient interaction in Shahid Faghihi hospital", which was approved (No. 1069353) at Shiraz University. The study was developed to increase knowledge and does not seek to question the efforts of the physicians who are working around the clock to serve patients in this hospital. The researchers would like to thank all of the participants in the study. In addition, the authors would like to thank the research consulting center of Shiraz University of Medical Sciences and their assistant for editing this article.

\section{References}

1. Dreher J. The Symbol and the Theory of the Life-World: "The Transcendences of the Life-World and Their Overcoming by Signs and Symbols". J Hum Stud. 2003;26(2):141-63. doi: 10.1023/a:1024014620368.

2. Sapolsky A. Relationship between Patient-Doctor Compatibility, Mutual Perception, and Outcome of Treatment. J Abnorm Psychol. 1965;70:70-6. [PubMed: 14271293].
3. Roter D, Hall JA. Doctors talking with patients/patients talking with doctors: improving communication in medical visits. US: Praeger Westport, CT; 2006.

4. Friedman HS. Nonverbal Communication Between Patients and Medical Practitioners. J Soc Issues. 1979;35(1):82-99. doi: 10.1111/j.15404560.1979.tb00790.x.

5. Sadati AK, Iman MT, Lankarani KB. Medical paraclinical standards, political economy of clinic, and patients' clinical dependency; A critical conversation analysis of clinical counseling in South of Iran. Int J Community Based Nurs Midwifery. 2014;2(3):157-68.

6. Sadati AK, Bagheri Lankarani K, Enayat H, Kazerooni AR, Ebrahimzadeh S. Clinical paternalistic model and problematic situation: a critical evaluation of clinical counseling. I Health Sci Surveillance Syst. 2014;2(2):78-87.

7. Parsons T. The Social System. England: Routledge; 2013.

8. Marcus GE, Fischer MM. Anthropology as cultural critique: An experimental moment in the human sciences. US: University of Chicago Press; 1999.

9. Singer M. The coming of age of critical medical anthropology. Soc Sci Med. 1989;28(11):1193-203. [PubMed: 2660276].

10. Scambler G, Britten N. Habermas, critical theory and health. 8. US: Routledge; 2001. System, lifeworld and doctor-patient interaction.

11. Foucault M. The Birth of the Clinic, Archeology of Medical Perception. London: Routledge; 2012

12. Davis MS. Variation in patients' compliance with doctors' orders: medical practice and doctor-patient interaction. Psychiatry Med. 1971;2(1):31-54. [PubMed: 5141549].

13. Thomas J. Doing critical ethnography. Thousand Oaks, CA: Sage; 1993.

14. Carspecken PF. Critical Ethnography in Educational Research: A theoretical and practical guide Routledge. New York: Routledge; 1996.

15. Sandelowski M. Rigor or rigor mortis: the problem of rigor in qualitative research revisited. ANS Adv Nurs Sci. 1993;16(2):1-8. [PubMed: 8311428].

16. Humble AM. Technique triangulation for validation in directed content analysis. Int J Qualitative Method. 2009;8(3):34-51.

17. American Sociological Association. . Code of ethics. US: ERIC Clearinghouse; 1984.

18. World Medical Association. . World Medical Association Declaration of Helsinki: ethical principles for medical research involving human subjects. J Postgrad Med. 2002;48(3):206-8. [PubMed: 12432198].

19. Mishler EG. The Discourse of Medicine: Dialectics of Medical Interviews. New Jersy: Albex Publishing Corporation; 1984.

20. Silverman D. Communication and medical practice: Social relations in the clinic. London: Sage Publications Ltd; 1987.

21. Atkinson P, Atkinson PA. Medical talk and medical work. London: Sage; 1995.

22. Barry CA, Stevenson FA, Britten N, Barber N, Bradley CP. Giving voice to the lifeworld. More humane, more effective medical care? A qualitative study of doctor-patient communication in general practice. Soc Sci Med. 2001;53(4):487-505. [PubMed: 11459399].

23. Sadati AK, Iman MT, Bagheri Lankarani K, Derakhshan S. A critical ethnography of doctor-patient interaction in southern Iran. Indian Med Ethics. 2016;1(3):147-55. [PubMed: 27474695].

24. Kalateh Sadati A, Tabei SZ, Ebrahimzade N, Zohri M, Argasi H, Bagheri Lankarani K. The paradigm model of distorted doctor-patient relationship in Southern Iran: a grounded theory study. J Med Ethics Hist Med. 2016;9:2. [PubMed: 27390617].

25. Hall JA, Roter DL, Katz NR. Meta-analysis of correlates of provider behavior in medical encounters. Med Care. 1988;26(7):657-75. [PubMed: 3292851].

26. Bensing J. Doctor-patient communication and the quality of care. Soc Sci Med. 1991;32(11):1301-10. [PubMed: 2068614].

27. Tabrizi JS. Quality of delivered care for people with type 2 diabetes: a new patient-centred model. J Res Health Sci. 2009;9(2):1-9. [PubMed: 23344165]. 